\title{
Premières observations sur les capacités de rhizogénèse adventive du chêne vert (Quercus ilex L.)
}

\author{
Michèle L'HELGOUAL'CH, H. ESPAGNAC \\ Faculté des Sciences, Laboratoire de Biologie végétale expérimentale, \\ 33, rue Louis-Pasteur, F 84000 Avignon
}

\begin{abstract}
Résumé
Les boutures de chêne vert sont de moins en moins rhizogènes au fur et à mesure du vieillissement de la plante sur laquelle elles sont prélevées. De 70 p. 100 chez les jeunes sujets, nous passons à 6 p. 100 d'enracinement lors du bouturage de fragments pris sur de vieux individus. Cette diminution se fait brutalement et précocement puisque pour des boutures provenant de chênes de 4 mois l'enracinement n'est plus que de 30 p. 100 et cela quel que soit le niveau de prélèvement. Chez les jeunes plantes de 1 mois les boutures apicales sont plus rhizogènes que les basales. Les rameaux axillaires, obtenus après décapitation de la tige principale de chêne de 1 mois, bouturés en entier donnent de meilleurs résultats que la tige principale. Cette supériorité des axillaires se retrouve lors du bouturage des rejets de souche, où seuls les rameaux secondaires sont capables de produire quelques racines.
\end{abstract}

Mots clés : Rhizogénèse adventive, bouturage, Quercus ilex $L$.

\section{Introduction}

La multiplication végétative des ligneux arborescents permet, dans certains cas au moins, la conservation et la multiplication des génotypes intéressants (KoRMANICK \& BRown, 1974). La méthode la plus usitée par les sélectionneurs consiste à provoquer par le bouturage de fragments de tige la formation de racines adventives; or dans de nombreux cas, le bouturage des arbres devient plus difficile à obtenir au fur et à mesure de leur vieillissement (Gardner, 1929 ; Thimann \& Delisle, 1939 ; Franclet, 1969).

L'intérêt suscité ces dernières années par la revalorisation des forêts méditerranéennes nous a conduit à tester dans ce domaine quelques espèces du genre Quercus. La présente note rend compte des premiers résultats que nous avons obtenus chez le chêne vert. Le bouturage est effectué à partir de fragments de tiges feuillées provenant d'une part de jeunes plants, issus de semis effectués en serre, d'autre part, de pousses prélevées sur des arbres ayant une vingtaine d'années ${ }^{(i)}$, enfin de fragments d'axes de rejets de souche.

(1) L’âge des individus aduftes utilisés dans nos expériences a été évalué par la technique des carottages. 


\section{Matériel}

Nous considérerons successivement : le matériel végétal, les conditions du bouturage, les méthodes d'observation et de mesures.

\subsection{Le matériel végétal}

Des glands récoltés en octobre $(1982,1983,1984)$ sur des chênes truffiers sont conservés au froid. Chaque mois, cent glands décortiqués sont mis à germer en serre dans un mélange tourbe et terreau. Cette technique nous permet au moment du bouturage ( 7 mois après la date du $1^{\text {er }}$ semis) de: travailler avec des individus d'âges différents. Les plantes obtenues sont cultivées en serre à une température qui n'est jamais inférieure à $10^{\circ} \mathrm{C}$ et en jours longs de 16 heures (lumière d'appoint).

Les individus âgés ont tous plus de 20 ans et proviennent d'une population localisée à Venasque (Vaucluse). Les fragments pour bouturage correspondent à la dernière pousse réalisée par le rameau considéré. Quant aux rejets de souche, ils sont issus de recépages effectués dans trois stations différentes: l'une dans le massif du Lubéron (coupe réalisée en septembre 1982), une autre à Venasque (Vaucluse ; coupe en mars 1983) ; enfin, une dernière à Blauvac (Vaucluse ; coupe en mars 1984). Nous disposons ainsi de rejets d'âges différents au moment des prélèvements qui s'effectuent au même moment.

\subsection{Les conditions de bouturage}

Les conditions de bouturage sont choisies en fonction des résultats de certains de nos prédécesseurs pour d'autres espèces (Cornuz, 1960 ; Franclet, 1976 ; Kohstall, 1978) et d'un certain nombre de nos observations préliminaires (L'Helgoval'CH, 1985).

Tous les fragments, avant d'être mis en culture, subissent un prétraitement de leur base dans du talc contenant un produit commercial rhizogène dont la concentration en A.I.B. est de 0,56 p. 100 et un fongicide (benomyl). Ils sont ensuite placés dans des bacs emplis de tourbe pure. Pour les boutures de jeunes chênes, la température du substrat est maintenue entre 25 et $35^{\circ} \mathrm{C}$ à l'aide d'une résistance électrique. Tous les bacs sont ensuite placés sous un tunnel à brouillard. L'humidification des boutures est assurée par une nébulisation intermittente se déroulant de $5 \mathrm{~h}$ à $22 \mathrm{~h}$, à raison de 1 demi-heure par heure. La température varie en moyenne de $25^{\circ} \mathrm{C}$ le jour à $10^{\circ} \mathrm{C}$ la nuit ; toutefois, durant les périodes les plus chaudes, la température en milieu de journée peut s'élever jusqu'à $45^{\circ}$. Toutes les boutures sont maintenues en jour long de 16 heures.

\subsection{Observations et mesures}

Les observations sont effectuées après 2 ou 3 mois de culture sous un tunnel à brouillard. Tous les fragments sont alors prélevés et ceux possédant au moins une racine sont comptabilisés. Les résultats sont évalués en pourcentage. Ces derniers sont ensuite comparés statistiquement (voir test en annexe). 


\section{Résultats}

Nous envisagerons essentiellement les résultats fournis par la tige principale et les rameaux latéraux de jeunes individus issus de semis. Ceux obtenus à partir de fragments de pousses prélevés sur arbres âgés et rejets de souches seront simplement évoqués.

\subsection{Le bouturage de fragments pris sur la tige principale de jeunes individus}

On sait que la tige de jeunes individus issus de semis et cultivés en serre est le plus souvent monocaule jusqu'à l'âge de 12 mois; elle est constituée par des pousses successives, résultats de la "croissance par vague ». Le nombre de ces pousses est d'autant plus important en moyenne que la plante est plus âgée (FÉraud, 1985).

L'expérience a consisté à bouturer la tige principale de plantes âgées de $1,2,3,4$, 7,12 et 24 mois. Pour chaque catégorie d'âges, une vingtaine d'individus sont prélevés et à partir de chaque tige, sectionnée juste au-dessus des cotylédons, sont constitués 2 lots de boutures. Le lot 1 comprend les parties basales sectionnées au-dessus des 2 ou 3 premiers nouds feuillés. Le lot 2 se compose des parties apicales débarrassées du bourgeon terminal et comportant uniquement les 3 ou 4 derniers nouds feuillés. Sur chacune de ces boutures ne sont conservées que les deux dernières feuilles étalées. Cette expérience a été réalisée à trois reprises. Les prélèvements et bouturages ont été effectués au mois de mars des années 1982, 1983 et 1984.

Dans un premier temps, les résultats obtenus pour les parties basales et apicales sont regroupés. Ceux-ci, obtenus pendant les trois années, pouvant être considérés après vérification comme similaires (ex. : plantes de 2 mois : 77 p. 100, 61 p. 100, 52 p. 100 ; plantes de 3 mois : 74 p. 100,81 p. 100,63 p. 100 ; plantes de 4 mois : 38 p. 100, 32 p. 100,22 p. 100 ; plantes de 7 mois : 15 p. 100,18 p. 100,20 p. 100) permettent d'établir un pourcentage moyen de fragments racinés pour chaque classe d’âges. Ces différents pourcentages sont représentés par le graphique de la figure 1. Immédiatement, on observe que les fragments prélevés sur des plantes de 1,2 et 3 mois s'enracinent dans de bonnes proportions, puisqu'on obtient environ 70 p. 100 de réussite. Une comparaison des valeurs respectives $(67,62,72$ p. 100) montre qu'elles ne sont pas significativement différentes. Par contre, parmi les boutures de plantes de 4 mois, 33 p. 100 seulement se sont enracinées. Ce résultat témoigne d'une chute considérable du pouvoir rhizogène, le plus étonnant dans cette baisse étant la brutalité avec laquelle le phénomène se produit. Par la suite, la diminution se poursuit pour atteindre $18 \mathrm{p}$. 100 à 7 mois et enfin à peine $6 \mathrm{p} .100$ pour des fragments prélevés sur des arbres âgés de plus de 20 ans. Dans ce dernier cas, l'enracinement n'est possible que si l'apex des fragments bouturés est en activité au moment du prélèvement. On retrouve donc chez le chêne vert une action stimulatrice de la rhizogénèse adventive en provenance des bourgeons terminaux en activité comparable à celle observée chez les boutures de vignes par Julliard (1963) et FaVRe \& Médard (1969).

Si maintenant sont envisagés séparément les résultats fournis par les parties basales et apicales des jeunes plants de 1 à 4 mois (fig. 2), on constate des pourcentages voisins pour les 2 lots de boutures concernant les plantes de 2 à 4 mois. La chute observée à 4 mois s'effectue aussi bien pour les parties basales $(31 \mathrm{p}$. 100) que pour les 


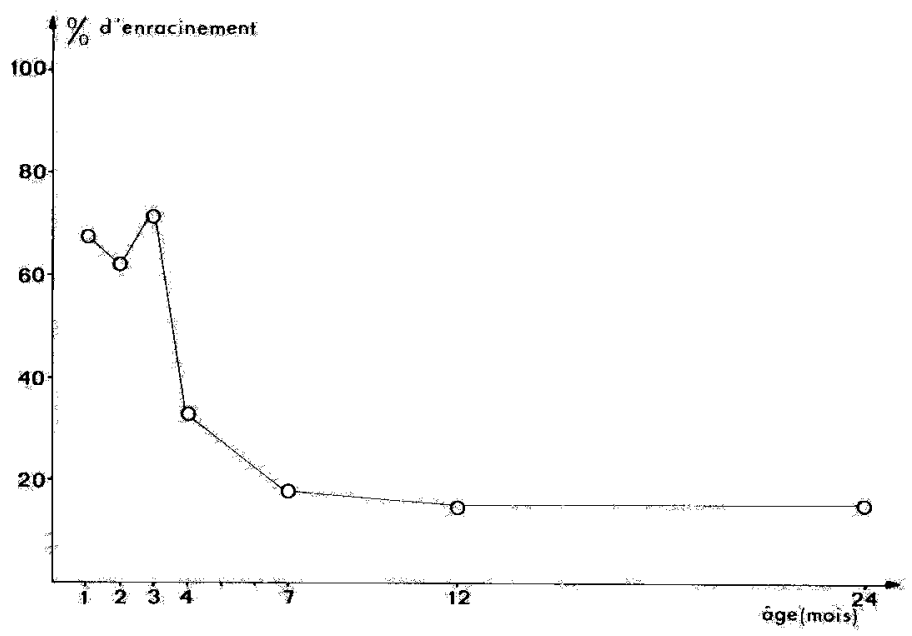

FIG. 1

Enracinement en fonction de l'âge du plant bouturế. Rooting according to the age of the plant used.

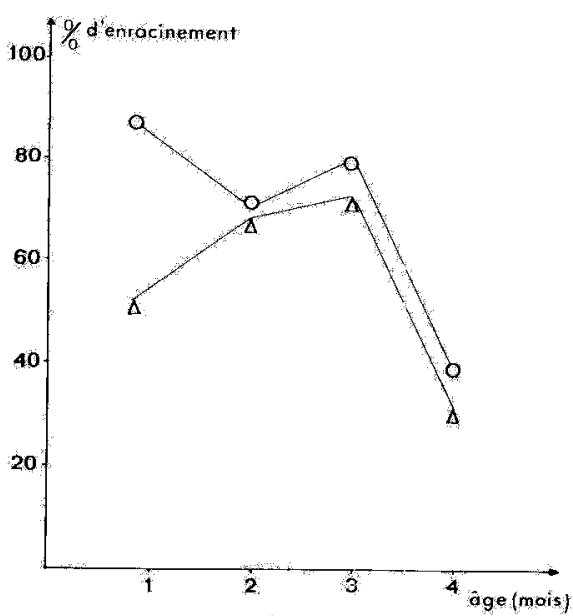

Fir. 2

Enracinement obtenu pour les parties apicates ( $0-0$ ) et basales $(\Delta \Delta)$ en fonction de lâge du plant bouture apres 2 mois de culture sous tunnel.

Rooting for apical $(\mathrm{O}-\mathrm{O}$ ) and basal $t \Delta \mathrm{\Delta}$ ) parts according to the age of the plant used after 2 months under mistsystem. 
parties apicales (38 p. 100). Par contre, pour les individus âgés de 1 mois, les fragments basaux s'enracinent à 52 p. 100 et les fragments terminaux à $87 \mathrm{p} .100$. Cette différence montre de façon significative que les parties basales de la tige principale s'enracinent moins bien que les parties terminales. Ce résultat, contraire à ce qui est classiquement admis (M.K. Chaudhri, 1966; Nozeran et al., 1982), avait déjà été obtenu au laboratoire lors du bouturage des parties basses de la tige de très jeunes Lauriers roses (BACHIR, 1983): Tout se passe comme si, chez les espèces en question, émanait du système racinaire une influence inhibitrice de la rhizogénèse adventive, capable de persister même après sa suppression ; cet effet négatif se ferait d'autant plus ressentir que la plante est plus jeune.

\subsection{Bouturage des rameaux latéraux de jeunes individus}

Après avoir testé les capacités d'enracinement adventif de la tige principale de jeunes individus, il nous a semblé intéressant d'évaluer celles des rameaux latéraux, en fonction en particulier de leur niveau d'insertion. Dans cette expérience, de jeunes chênes verts de 1 mois sont utilisés; à cet âge, l'appareil aérien des individus est monocaule, le développement de rameaux latéraux nécessite une décapitation de la tige principale qui supprime l'inhibition corrélative exercée par le bourgeon terminal. Des ablations plus ou moins importantes de l'axe principal sont réalisées afin d'obtenir la croissance de rameaux latéraux insérés à des hauteurs diverses. Dans le premier lot, 20 plantes subissent l'ablation du bourgeon terminal (fig. 3a) provoquant ainsi le développement de 1 à 2 rameaux axillaires subapicaux. Le second lot est constitué par 20 plantes décapitées au-dessous des deux derniers nœuds feuillés (fig. 3b), se sont alors le plus souvent deux axes médians qui se développent. Dans le troisième lot enfin, 20 épicotyles sont sectionnés juste au-dessus de l'insertion des cotylédons (fig. 3c), ce qui entraîne le démarrage des bourgeons situés à leur aisselle. Les rameaux latéraux ainsi produits sont prélevés dans leur intégralité et bouturés 17 jours après l'arrêt de leur première vague de croissance. Dans le même temps, un quatrième lot est constitué d'axes principaux de 20 plantes de 1 mois n'ayant subi aucune décapitation (fig. $3 \mathrm{~d}$ ). Les boutures des divers lots sont traitées comme précédemment et placées sous tunnel à brouillard. Les observations sont réalisées 30,47 et 61 jours après bouturage. Les résultats sont consignés dans le tableau 1.

\section{TABLEAU 1}

Enracinement des divers axes secondaires et des axes principaux de plantes de 1 mois en fonction du temps passé sous tunnel.

Rooting of the various secondary ramifications and of the main ramification for one month-old plants according to the duration of their staying under mist-system.

\begin{tabular}{|c|c|c|c|}
\hline Rang, rameau $\begin{array}{r}\text { Temps en jours } \\
\text { observés }\end{array}$ & 31 & 47 & 61 \\
\hline 10 rameaux subapicaux $\ldots \ldots \ldots$ & $70 \%$ & $80 \%$ & $80 \%$ \\
\hline 22 rameaux médians & $77 \%$ & $80 \%$ & $92 \%$ \\
\hline 12 rameaux cotylédonaires. & $33 \%$ & $66 \%$ & $66 \%$ \\
\hline 20 tiges principales témoins & non relevé & $25 \%$ & $25 \%$ \\
\hline
\end{tabular}


A la fin de l'expérience, c'est-à-dire 61 jours après le bouturage, on constate que les rameaux latéraux, obtenus après suppression de la dominance apicale, sont plus rhizogènes que la tige principale quel que soit leur niveau d'insertion ; même les axes cotylédonaires (66 p. 100) sont significativement plus performants. Il faut signaler ici qu'un résultat comparable a été obtenu au niveau des rejets de souches chez lesquels seuls les rameaux latéraux se sont révélés capables de produire des racines adventives et encore seulement dans 8 p. 100 des cas (L'Helgoual'ch, 1985). Si l'on s'intéresse maitenant aux résultats obtenus 31 jours après le début de l'expérience, on observe que les rameaux cotylédonaires s'enracinent à 33 p. 100 et réagissent semble-t-il moins bien que les deux autres catégories de rameaux $(70$ p. 100 et 77 p. 100). Toutefois, étant donné que cette différence tend à s'estomper avec le temps, on est en droit de penser qu'elle traduit seulement une réponse plus lente. Cette interprétation est d'ailleurs confirmée par les observations consignées dans le tableau 2. Celles-ci mettent en évidence que tous les fragments, quelle que soit leur origine s'enracinent avec la même intensité $(2,07 ; 2,3 ; 2,5$ racines) mais que, au moment des observations, les racines produites par les rameaux cotylédonaires sont courtes $(5 \mathrm{~mm})$ et non ramifiées alors que celles des autres rameaux sont nettement plus longues $(50$ et $60 \mathrm{~mm})$ et ramifiées. Néanmoins, il ne faut pas oublier que nous travaillons sur un petit nombre de rameaux et qu'il y a lieu d'être prudent quant aux conclusions.

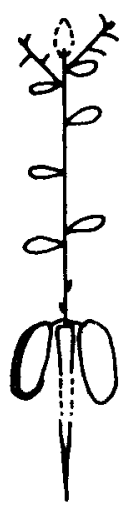

a

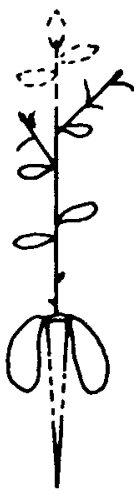

b

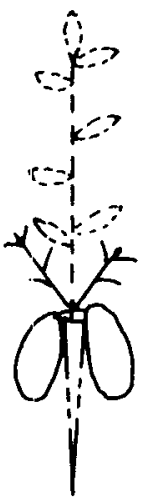

C

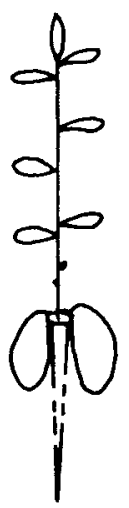

d

FIG. 3

Décapitation de l'axe principal à divers niveaux (plants de 1 mois).

Section of the main stem on different levels (one month-old plants).

a : Bourgeon terminal supprimé. Développement des $\mathrm{A}_{2}$ subapicaux.

Terminal bud taken off. Development of $A_{2}$ close to the apical bud.

$\mathrm{b}$ : Bourgeon terminal et les deux derniers nœuds supprimés. Développement des $\mathrm{A}_{2}$ médians.

Terminal bud and two last nods taken off. Development of median $A_{2}$.

c: Coupe au-dessus des cotylédons. Développement des $\mathrm{A}_{2}$ cotylédonaires.

Section above the cotyledons. Development of the $A_{2}$ sited on the cotyledon axil.

d : Axe principal non décapité - Uncut main stem. 
Tableau 2

Tableau donnant pour chacune des catégories de rameaux latéraux le nombre moyen de racines par fragment bouturé, ainsi que leur longueur après 30 jours de culture sous tunnel.

Table showing for each lateral ramification the average number of root per cutted extract, and their length after 30 days under mist-system.

\begin{tabular}{l|c|c|c|c}
\hline & $\begin{array}{c}\% \\
\text { de fragments } \\
\text { racinés }\end{array}$ & $\begin{array}{c}\text { Nombre } \\
\text { moyen } \\
\text { de racines } \\
\text { par fragment }\end{array}$ & $\begin{array}{c}\text { Longueur } \\
\text { moyenne } \\
\text { des racines } \\
\text { Ramm) }\end{array}$ & $\begin{array}{c}\text { Présence } \\
\text { de racines } \\
\text { secondaires }\end{array}$ \\
\cline { 2 - 5 } Rameaux médians $\ldots \ldots \ldots \ldots \ldots \ldots$ & $70 \%$ & 2,07 & 50 & oui \\
\hline Rameaux cotylédonaires $\ldots \ldots \ldots \ldots \ldots$ & $33 \%$ & $2, \ldots$ & 60 & oui \\
\hline \hline
\end{tabular}

\section{Discussion et conclusions}

La baisse du pouvoir rhizogène adventif, qui est un phénomène classiquement observé chez les ligneux arborescents, se produit chez le chêne vert de façon très précoce puisqu'elle s'observe dès l'âge de 4 mois. De plus, la baisse de l'aptitude au bouturage se manifeste, chez cette espèce, d'une façon particulièrement brutale ; à l'âge de 3 mois, 70 p. 100 en moyenne des boutures réalisées s'enracinent alors qu'à l'âge de 4 mois, la performance n'est plus que de $30 \mathrm{p}$. 100. Les facteurs externes ne semblent pas intervenir, du moins de façon déterminante, puisque toutes les boutures ont été cultivées dans les mêmes conditions. La structure anatomique ne peut pas non plus être mise en cause, contrairement à ce qui se passe chez le chêne rouvre (KazandJian, 1977) car les différences observées entre les fragments prélevés sur des individus de 3 ou 4 mois sont très légères et ne peuvent en aucun cas expliquer des performances aussi éloignées. Quoi qu'il en soit, l'importance et la brutalité de la diminution du pouvoir rhizogène adventif chez Quercus ilex créent une situation expérimentale intéressante que nous comptons bien continuer à exploiter.

D'autres résultats obtenus chez de jeunes plantes de 1 mois nous paraissent intéressants. Ainsi, chez ces dernières, la réussite du bouturage de la partie proximale de la tige est toujours plus faible que celle de la partie distale, ce résultat contraire aux notions classiquement admises selon lesquelles les portions les plus proches de l'appareil racinaire sont plus facilement rhizogènes a déjà été obtenu avec une autre plante : Nerium oleander (BACHIR, 1983). Nous supposons, d'après ce résultat qu'il existe un effet inhibiteur de la rhizogénèse adventive émanant du pivot racinaire principal et capable de continuer à se manifester après la suppression de ce dernier. Cette influence négative ne se fait sentir que chez de très jeunes plantes et semble disparaître au cours du développement ou se transformer en une influence positive. De tels exemples de modifications de l'influence d'un organe au cours de son développement sont déjà 
connus chez les végétaux (Neville, 1970). Cet effet inhibiteur, émanant du pivot racinaire semble se retrouver lors du bouturage des rameaux latéraux, ceux insérés aux niveaux élevés fournissent des résultats meilleurs que les axes cotylédonaires.

Quant aux résultats obtenus à partir des pousses prélevées dans la cime d'arbres âgés, ils permettent de constater une stabilisation de la rhizogénèse adventive à un niveau très bas $(6 \mathrm{p} .100)$; et ils mettent en évidence le rôle joué par l'état du bourgeon terminal au moment de la mise en bouture. Les tentatives de rajeunissement de notre matériel par recépage ont été décevantes, aussi nous tentons à l'heure actuelle de transférer nos expériences dans le domaine de la culture in vitro, ce qui devrait fournir d'utiles renseignements.

Reçu le 25 juillet 1986.

Accepté le 4 février 1987.

\title{
Remerciements
}

Nous tenons à remercier les chercheurs du Laboratoire de Botanique Historique et de Palynologie (Faculté des Sciences Saint-Jérôme, Marseille) qui nous ont aidé à évaluer l'âge des individus adultes par la technique de carottages.

\author{
Summary \\ First observations on adventive rhizogenesis capacities of Quercus ilex $L$.
}

Rooting of stem fragments of Quercus ilex L., from young plants seedling culture in a greenhouse and from adult trees, is studied. Experimentation is made under mist-system - substrat being kept under $25^{\circ} \mathrm{C}$ - for the young plants cuttings. The rate of successful propagation by cuttings gets up to $70 \mathrm{p}$. 100 for 3 months old plants but decreases down to $15 \mathrm{p}$. 100 for 24 months plants and 6 p. 100 for old trees (fig. $\mathrm{n}^{\circ} 1$ ). Concerning this last case, rooting is possible only if buds are active when taken off. Two facts can be noted: firstly, rhizogenetic adventive capacities of our species decrease very early (4 months-old plants), secondly, it is also very sudden as from $70 \mathrm{p}$. 100 rooting for 3 months-old plants, we get down to $30 \mathrm{p} .100$ for 4 months-old plants. The withdrawal level acts upon the cutting of one month-old plants (fig. 2) : the higher part gives better results than the lower part. This enables us to think that there is a negative influence out of rooting-system which remains in spite of the withdrawal and only concerns the very young plants.

Rooting of secondary axis, obtained on one month-old plants after decapitation of the main stem (fig. 3), is better than rooting of main stem. Similar results are observed with stump shoots, secondary axis only can produce a few rooting fragments ( 8 p. 100).

Key words: Adventive rhizogenesis, cuttings, Quercus ilex $L$. 


\section{Annexe}

\section{Comparaison de deux pourcentages}

1. Pour des échantillons à effectifs faibles $(n \leqslant 100)$

$$
\mathrm{p}\left(\frac{\mathrm{a} 1}{\mathrm{n} 1} ; \frac{\mathrm{a} 2}{\mathrm{n} 2}\right)=\frac{C_{\mathrm{n} 1}^{\mathrm{a}}\left(C_{\mathrm{n} 2}^{\mathrm{a} 2}\right.}{C_{\mathrm{n} 1}^{\mathrm{a}}+C_{\mathrm{n} 2}^{\mathrm{a} 2}}=\frac{\mathrm{n} 1 ! \mathrm{n} 2 !(\mathrm{a} 1+\mathrm{a} 2) !(\mathrm{n} 1+\mathrm{n} 2-\mathrm{a} 1-\mathrm{a} 2) !}{(\mathrm{n} 1+\mathrm{n} 2) ! \mathrm{a} 1 !(\mathrm{n} 1-\mathrm{a} 1) ! \mathrm{a} 2 !(\mathrm{n} 2-\mathrm{a} 2) !}
$$

si $\mathrm{P}<0,05, \mathrm{q} 1-\mathrm{q} 2$ significatif

$\mathrm{n} 1=$ effectif du premier échantillon, $\mathbf{q} 1$ sa fréquence

$\mathrm{a} 1=$ nombre d'individus présentant le caractère $\mathrm{q} 1$

n2 = effectif du deuxième échantillon, q2 sa fréquence

$\mathrm{a} 2=$ nombre d'individus présentant le caractère $\mathrm{q} 2$

2. Pour des échantillons à effectifs élevés $(n \geqslant 100)$

Calcul de la variance:

$$
\mathrm{S}^{2} \mathrm{~d}=\mathrm{Qe}(1-\mathrm{Qe}) \frac{1}{\mathrm{n} 2}+\frac{1}{\mathrm{n} 1}
$$

au seuil 95 p. $100, \mathrm{t}=1,96 \simeq 2$

si $\mathrm{t}<2, \mathrm{q} 1-\mathrm{q} 2$ n'est pas significative

$2,6>t>2, q 1-q 2$ est significative

$$
\mathrm{t}=\frac{\mathrm{q} 1-\mathrm{q} 2}{\mathrm{Sd}}
$$

\section{Références bibliographiques}

Bachir S., 1983. Contribution à l'étude de la rhizogénèse adventive chez le Laurier rose (Nerium oleander L.). Thèse de $3^{\mathrm{e}}$ cycle, Aix-Marseille III.

Cauvin S., Marien J.N., 1978. La multiplication végétative des Eucalyptus en France. C.R. Ass. For. Cell. (A.F.O.C.E.L.), Ann. Rech. Sylvic., 141-175.

CHAudhri M.K., 1966. Etude de quelques facteurs sur la Rhizogénèse chez les boutures ligneuses de plusieurs espèces du genre Prunus. Thèse de $3^{\mathrm{e}}$ cycle, Bordeaux.

Cornu D., Delran S., Garbaye J., Le Tacon F., 1977. Recherches des meilleures conditions d'enracinement des boutures herbacées de Chêne rouvre (Quercus petraea (M), Liebl.) et de Hêtre (Fagus sylvatica L.). Ann. Sci. For., 34, nº 1, 1-16.

Cornuz L.A., 1960. Brouillard artificiel et influence du substratum sur les boutures feuillées d'arbustes. Rev. Hort. Suisse, 3, 344-348.

Favre J.M., Medard R., 1969. Ontogénie des racines adventives chez la Vigne cultivée in vitro. Rev. Gen. Bot., 76, 455-467.

Feraud C., 1985. Quelques aspects de la croissance et du développement du Chêne vert (Quercus ilex L.) et premiers essais de culture in vitro. Thèse $3^{\mathrm{e}}$ cycle, Aix-Marseille III.

Franclet A., 1969. Propagation of conifers and other difficult species by cuttings. In proceed. end. F.A.O./U.F.R.O. World consult. for breeding, Washington F.O./F.T.B., 11. 4-11.

FRANClet A., 1976. Recherches des conditions favorables au bouturage du pin maritime. A.F.O.C.E.L., 75 . 
Gardner F.E., 1929. The relation between tree age and the rooting of cuttings. Proc. Am. Soc. Hort. Sc., 26, 101-104.

Julliakd B., 1963. Influence du bourgeon sur la rhizogénèse des boutures de vigne (Vitis vinifera L.). Note présentée par Gautheret, C.R. Acad. Sc. Paris, 257, 3200-3203.

Kazandian B., 1977. La multiplication végétative du Chêne par bouturage. Mémoire de $3^{e}$ année d'E.N.I.T.E.F., Domaine des Barres, Nogent-sur-Vernisson.

Kohstall M., 1978. La multiplication des végétaux ligneux sous tunnel plastique. Plasticulture $\mathrm{n}^{\mathrm{o}} 37,45-52$.

Kormanik P.P., Brown C.L., 1974. Vegetative propagation of some selected forest species in the south castern United States. N. Z. J. Forest. Sci., 4, n' 2, 228-234.

L'Helgoual'ch M., 1985. La rhizogénèse adventive chez le Chêne vert (Quercus ilex L.). Thèse $3^{e}$ cycle, Aix-Marseille III.

Martin B., Quiliet G., 1974. Bouturage des arbres forestiers au Congo. Revue Bois et Forêts des Tropiques, $\mathrm{n}^{\text {os }} 154$ à 157.

Nevicte P., 1970. Morphogénèse chez Gleditsia triacanthos L. V. Influence des feuilles de divers âges sur le développement des feuilles sus-jacentes. Rev. Gen. Bot., 77, 387-407.

Nozeran R., Ducreux G., Rossignol-Bancilhon L., 1982. Réflexions sur les problèmes de rajeunissement chez les Végétaux. Bul. soc. Bot.. 129, Lettres botaniques L., 107-126.

Thimann K.V., Delisi.e A.L., 1939. The vegetative propagation of difficult plants. J. Arnold Arbor., vol. 20, 116-137. 